\title{
Use of Technological Diagnosis in Rail Vehicle Engines Maintenance
}

\author{
Jozef Ondriga $^{1^{*}}$, Slavomír Hrček ${ }^{1}$, František Brumerčík ${ }^{1}$ and Michal Lukáč ${ }^{1}$
}

${ }^{1}$ University of Žilina, Department of Design and Mechanical Elements, Žilina, Slovakia; Email: jozef.ondriga@fstroj.uniza.sk, slavomir.hrcek@fstroj.uniza.sk,frantisek.brumercik@fstroj.uniza.sk, michal.lukac@fstroj.uniza.sk

\section{*Corresponding Author: Jozef Ondriga}

Received: 10 June 2021; Revised: 31 August 2021; Accepted: 18 November 2021; Published: 30 November 2021

Abstract: The paper aims to classify the maintenance of internal combustion engines in 812 series diesel multiple units, to analyse operating failures, and to identify methods and procedures for determining their current mechanical condition. The paper specifies the required procedures and methods for maintenance starting from the development of the DMU (diesel multiple units) 812 series to the present. It also focuses on understanding how diagnosis and control sensors are attached in individual cable harnesses. A protocol for diagnosing combustion engine defects is proposed in the final part of the paper. A part of the paper is a proposed list of codes for individual faults to facilitate the work. Another method proposed within this paper is the definition and attachment of individual sensor connections on the engine in the EDC (Electronic Diesel Control) $\mathrm{M}(\mathrm{S}) 5$ cable harness. Faults leading to engine stop are identified.

Keywords: Diagnosis, maintenance, MAN-cats, MIREL 812, EDC

\section{Introduction}

ZSSK (Železničná spoločnost' Slovensko), a.s. currently operates 147 DMUs powered by a total of 186 MAN engines. The paper focuses on the use of technological diagnosis in the maintenance of MAN internal combustion engines of the rail vehicles series 812, which consist of 64 parts in total. The MAN-cat I diagnosis was used to diagnose faults on these engines. As MAN specializes mainly in on-road vehicles, diagnosis for separately supplied engines of the series 812 is not as correct and user-friendly as for the diesel multiple units of the series 813 and 861 .

For the purposes of the paper, description and understanding of the relationship of the MAN engine with other diesel multiple units of the series 812 (RS MIREL 812, cooling circuit BEHR, glow circuit) are required, as well as a proposal of a fault detection protocol, and design of a list of individual fault codes. 


\section{Data and Methods}

All measurements were taken with MAN-Cats III diagnostic on DMUs 812 series at the locomotive depot in Filakovo. For more accurate identification of possible engine faults that cannot be read on the information panels, fault conditions were simulated by disconnecting the individual connectors. To trigger the faults, it was necessary to start the engine and clear the EDC fault memory. After each disconnection of the connector, the fault and all the related information had to be written down, the entry needs to be cleared from the memory, and a systematic step-by-step procedure follows. First, the connectors of the EDC cable were disconnected, then the same procedure was followed for other cable harnesses.

\section{MAN D 2866 LUH 21 Engines with in-line Injection Pump Controlled by EDC M(S) 5} All measurements were taken on the MAN D 2866 LUH 21 engines. The DMU 812 series was developed using the MAN D 2866 LUH 21. The engine was mounted in the centre of the engine unit and suspended under the vehicle's floor on the vehicle's frame [1].

\section{Maintenance of MAN Combustion Engines in 812 Vehicles in the Locomotive Depot}

The MAN D 2866 LUH 21 combustion engine was selected as the basic model of the engine to consider the maintenance in the locomotive depot conditions. The maintenance of MAN internal combustion engines can be divided into three categories based on the warranty:

- after the production of the new DMU 812 series, under warranty,

- after the expiration of the car warranty under the terms of ZSSK, a.s.,

- as a separately supplied unit under warranty after overhaul (OVHL) for ZSSK, a.s..

Currently, the DMU 812 series drives are maintained according to MAN manufacturer's overhaul intervals for the engine type MAN D2866 LUH21. After a given mileage of the rail car, the range of diagnosis and repair interventions that must be undertaken is calculated at each interval $[2,3]$.

\subsection{Overview of MAN Defects in the 812 Series Vehicles}

MIREL 812 is a primary control system for the 812 series engine units. The internal combustion engine, starter and glow, traction gearbox, traction gearbox retarder, axle gearbox, and acoustic and optical fault signalling are all regulated by MIREL 812 [3]. It carries out continuous self-diagnosis and performs a practical test to ensure that all components of the control system and cooperating equipment on the rail vehicle are working properly. 


\subsection{Fault Signalling According to the MIREL 812 Manual}

The primary MIREL 812 diagnosis of the 812 series rail vehicle engine displays faults on the information panel of the control systems. In addition to the central diagnosis, each of the basic units of a rail vehicle has its own (more detailed) diagnosis including:

- EDC MS (5) internal combustion engine,

- VOITH Automatic traction gearbox,

- VZ1 train safety system,

- RM1 registration speedometer

Detected faults are displayed on the information panels of both driver's stations. In the case of MIREL RS 812, the detection of a fault is shown by the red "ERR" mark lighting up [4-6].

Faults of the MAN engine and glow are defined by a numerical code in the "OTHERS" menu column, along with their specification.

\section{Description of Cable Harnesses}

The engine cable harness includes the following connectors:

\subsection{EDC M(S) 5 Cable Harness}

The following connectors are used in the cable harness (Fig. 1):

1. engine speed sensor

2. auxiliary speed sensor

3. water temperature sensor from the engine heads

4. diesel temperature sensor (injection pump)

5. injection pump control rod sensor and control

6. air charge pressure sensor

7. first cylinder injector needle position sensor

8. connection connector for EDC cabling via junction box 


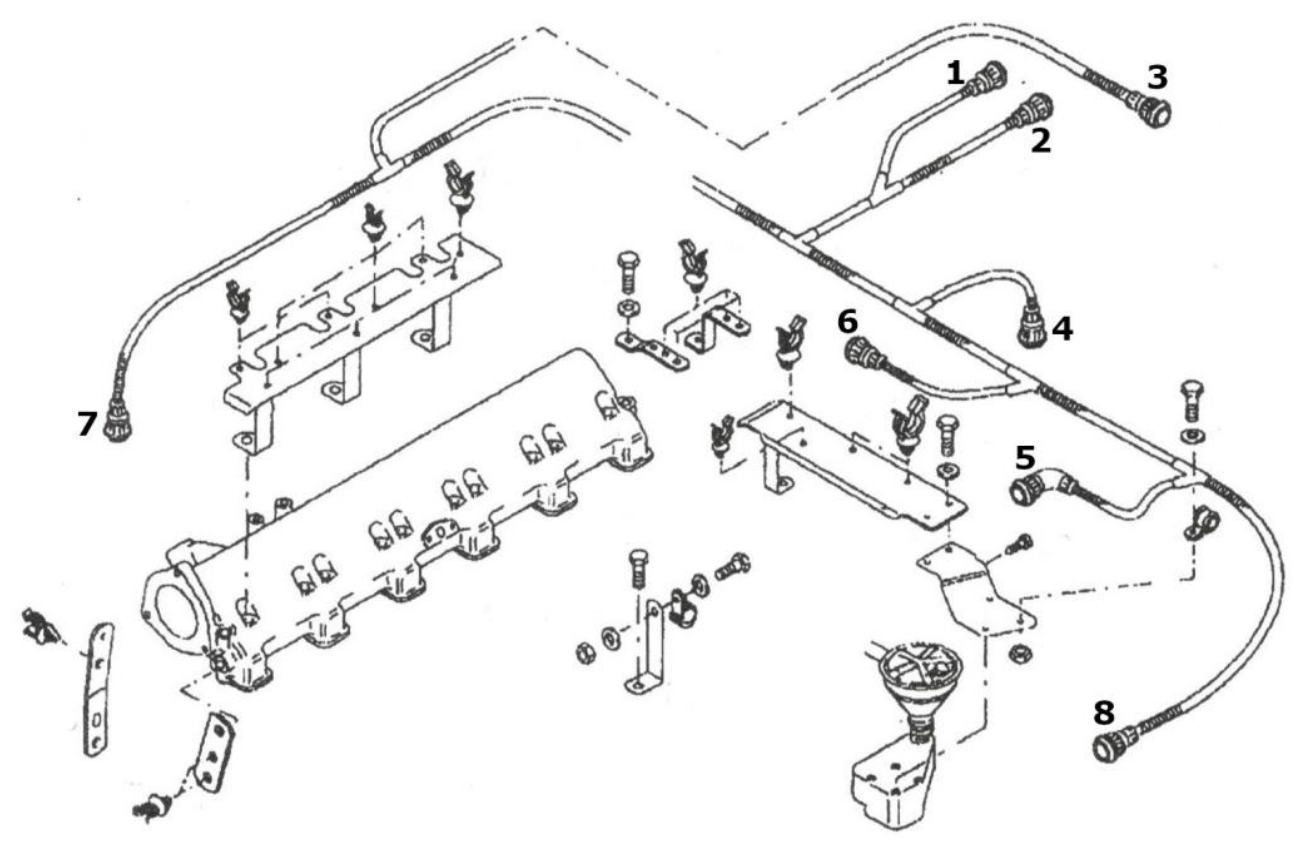

Fig. 1 EDC M(S) 5 Cable harness. Source: [5]

In addition to the EDC M(S) 5 cable harness, a motor head water temperature sensor (connector no. 3 ) is also attached to the MIREL 812 control system. The signals from this sensor are crucial for engine operation. If the coolant temperature reaches $100^{\circ} \mathrm{C}$, the RS MIREL 812 displays both visual and acoustic signal of a fault condition on the information panel [2,7]. If the temperature reaches $110^{\circ} \mathrm{C}$, the RS 812 disengages the traction gearbox and reduces the engine speed to idling. Until the engine has cooled down below this value, the rolling stock cannot be driven properly (if the driver permanently holds the reset button on the information panel and continues driving, there is a risk of serious engine damage).

The use of original complete MAN cabling in the design of the 812 Series DMUs was not possible. Some sensors are part of the DMU cable harnesses and are not attached to the EDC M(S) 5 , they have their own circuits.

\subsection{BEHR Cooling Cable Harness}

The air charge sensor is connected to the internal combustion engine cooling system. This sensor is fitted to the piping leading to the BEHR capacitor.

The combined BEHR radiator cools the engine coolant, engine charge air from the TBD, and the hydro-static oil used in the hydraulic movement of the radiator propeller. The signal of the sensor is fed to the BEHR cooling control module. This module detects the temperature of the engine coolant, receives a signal from the air charge sensor, and activates the solenoid that controls the speed of the cooling hydraulic engine. It is an isolated system, and if any signal is lost (due to temperature sensor failure), the cooling switches to emergency mode, and the cooling propeller 
operates at maximum speed. This system does not interfere with the internal combustion engine control system MIREL [8-10].

\subsection{Cable Harness for RS MIREL 812}

Two sensors are attached to this cable harness, an oil pressure sensor and a water temperature sensor in the engine block.

Oil pressure indicator is located at the bottom of the engine on the low numbers of the DMU 812 series. Higher numbers apply to a pressure sensor on the turbocharger. This sensor communicates the current engine oil pressure to the control system, which varies based on the engine temperature and rpm. When the pressure decreases to 1 bar, the RS 812 sends a visual and audible signal to report a fault. When the pressure decreases to $0.7 \mathrm{bar}$, the control system shuts down the engine [2,11].

The water temperature sensor is mounted on the top of the engine block. This sensor has only an information function for MIREL RS 812. It displays the engine temperature on the driver information panel starting at $40{ }^{\circ} \mathrm{C}$; it does not interfere with steering. It can be used in conjunction with MAN-cats I to measure the values of both temperatures and decide which one is inaccurate without having to disassemble them.

As the MAN-cats I diagnosis did not display any fault once they were removed, the water temperature sensor on the engine block and the oil pressure sensor are not attached to the EDC circuit. The MIREL 812 control unit is linked to both sensors.

\subsection{Glow}

The glow circuit is directly attached to MIREL 812, and its fault state is indicated both by audible and visual signal. The related blinking code is used to pinpoint the source of the problem. Faults in the glowing circuit are not registered in the EDC M(S) 5 memory.

\section{Fault Diagnosis}

Unlike the "Diagnosis, repair orders MIREL 812" manual, MAN engine faults with a numeric code (0 - 58) cannot be displayed in the "OTHERS" menu tab on the MIREL information screen. Only if MIREL 812 evaluates the fault as serious and "MULTIPLE FAULT" is displayed on the screen with the option to open this menu using the navigation button, the fault status can be displayed. Due to this shortcoming, neither the operator nor the maintenance personnel are able to find out what the engine fault is [12-15]. 


\subsection{Connection of MAN-cats I}

The WDI-2 communication interface is connected to the vehicle through the diagnostic socket in the vehicle's main substation.

When well-connected and with a good contact with the $\operatorname{EDC} \mathrm{M}(\mathrm{S}) 5$, the software would immediately provide navigation steps to access the fault memory as well as the diagnosis itself. Since the $\operatorname{EDC~M(S)~} 5$ is not connected to all engine functions (heating, oil pressure, and charge air temperature), its capabilities in comparison with road vehicles are limited to sensors which are directly attached to it (EDC M(S) 5 harness). MAN-cats I uses obsolete that did not come with a "fault decoder" with it; therefore, it is not possible to use the support of the software.

As a result, tests had to be performed directly on engine units, and "fault decoder" needs to be developed. The first measurement was taken on vehicle 812 033-9. The fault was defined in the fault book as follows: "The machine was operating at full speed; after a short time, it detected the MAN (R) fault, and the engine speed fell to idling." This indicates that the control system's reaction was visual and audible signalling the related MAN fault, resetting the fixed power rating, and disconnecting the traction gearbox $[2,16]$. The fault status menu could not be accessed via the information panel because it was not a case of multiple faults. The detected fault could be reset, and the vehicle could be driven again. From the $\operatorname{EDC~M(S)~} 5$ memory (Fig. 2), following information were read by connecting with MAN-cats I.

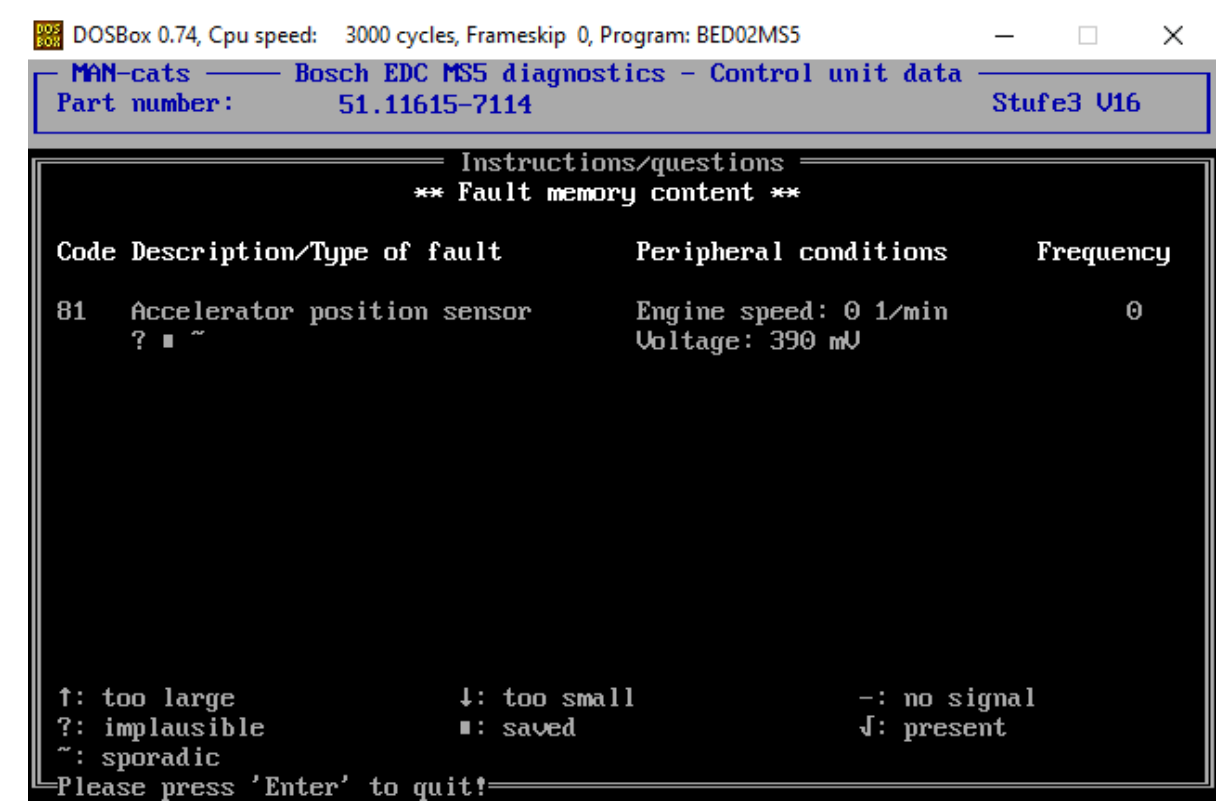

Fig. 2 EDC memory. Source: authors

Fault conditions were simulated by disconnecting individual connectors to detect more reliably emerging engine faults that could not be read on the information panels. The faults had to be triggered by starting the motor, and the EDC fault memory showed no fault recording. The record 
from the memory had to be removed after each connector disconnection and continued systematically step by step.

To track the records in EDC M(S) 5 of additional vehicles, MAN-cats I was used. Two types of EDC were detected on the vehicles, namely 51.11615-7203 and 51.11615-7114. The navigation key on the information panel was used to get to the "keys" display and the confirmation key to validate this feature using the "Diagnostic mode" of the MIREL RS 812 control system. The EDC power supply circuit was switched on and the detected faults in the vehicles were read using MAN-cats I in this manner. Table 1 shows the number of confirmed faults.

\section{Proposal of New Rational Procedures for Using Technical Diagnosis for MAN Engines and Proposal of Extended Diagnosis to 12-cylinder Engines}

To correctly diagnose defects, detailed knowledge of the vehicle at all levels is needed, including construction, vehicle regulation, interconnection, and coordination of independent structures. Without this information, it is impossible to determine the cause of the malfunction based solely on diagnostic evidence. This chapter deals with the discussion on how to detect and troubleshoot problems.

\subsection{Procedure for Fault Identification on the MAN D 2866 LUH 21 Engine in DMU 812 Series}

Following the arrival of vehicle 812 to the engine house, the protocol must be as follows:

- reading the repair book,

- MIREL RS 812 system check (indicated faults MAN (R), VOITH, multiple faults, etc.),

- switch the MIREL RS 812 to diagnostic mode and read faults from EDC M(S) 5 (if the workplace is equipped with MAN-cats III diagnosis),

- evaluate the information and determine the probable cause of the failure,

- determine the procedure and co-operation of individual professions in eliminating the defect,

- troubleshooting,

- clearing fault conditions and stationary testing of the vehicle,

- test drive, if necessary, recall of the operating conditions under which the fault occurred (if it is not possible to test the systems stationary).

Discovered cases where the engine stops while dealing with MAN-cats I and RS MIREL 812:

- failure of both speed sensors,

- failure of the sensor and control of the injection rod control,

- loss of power supply to the EDC cable from the on-board network,

- injector needle jam on the first cylinder, 
- too many pulses (errors) from the injector needle sensor on the first cylinder,

- critically low oil pressure in the MAN internal combustion engine. Pressure lower than 0.7 bar.

To assist in the identification of faults found by MAN-cats I a "Fault code-book" (Table 1) was produced. Specific fault codes are presented in the alphabetical order and translated into Slovak (see Table 1 below).

Table 1 "Fault code-book". Source. authors

\begin{tabular}{|c|c|}
\hline Fault code & Fault \\
\hline 11 & Fuel temperature sensor \\
\hline 32 & EEPROM Error R1 \\
\hline 38 & $\begin{array}{l}\text { SHUT-DOWN Routine not } \\
\text { completed }\end{array}$ \\
\hline 81 & Accelerator position sensor \\
\hline 84 & Engine speed sensor \\
\hline 85 & Charge air pressure sensor \\
\hline 87 & Water temperature sensor \\
\hline 88 & Road speed signal \\
\hline 96 & Microprocessor coupling \\
\hline $1 \mathrm{~A}$ & NBF Internal resistance faulty \\
\hline $1 \mathrm{~A}$ & NBF Too many pulses (faults) \\
\hline $1 \mathrm{~A}$ & $\begin{array}{c}\text { NBF Too few pulses (jammed } \\
\text { needle) }\end{array}$ \\
\hline $1 \mathrm{C}$ & Resistor array SG Pin 35 \\
\hline $1 \mathrm{~F}$ & CAN interface \\
\hline $8 \mathrm{~A}$ & Quantity regulator monitor \\
\hline $8 \mathrm{E}$ & Aux. engine speed sensor \\
\hline A7 & Resistor array SG Pin 54 \\
\hline
\end{tabular}

\subsection{Proposal for the Extension of Diagnosis on 12-Cylinder Engines}

MAN-cats III is a diagnostic tool for 12-cylinder engines. The MAN-cats III diagnosis is similar to the MAN-cats I diagnosis, but it is more advanced and user-friendly. Each fault is identified in detail. It also suggests potential remedy for the faults.

\section{Technical-Economic Evaluation of Proposals}

The proposed technique, a "fault decoder" with a Slovak translation and a simple separation of the connectors of individual sensors, would significantly reduce the amount of time necessary to 
diagnose a vehicle fault. It would allow easier sensor testing and a more precise identification of the fault's root cause. Moreover, it would also significantly reduce the workload. Determining the cause of error conditions would eliminate confusion and erroneous evaluation of the malfunction, which would result in reduced material costs and lengthy downtimes. This would directly influence the train schedule.

\subsection{Benefits of Using MAN-cats I Diagnosis}

The main financial benefit is the reduction in maintenance time and the elimination of inaccurate sensors and cable harnesses, which could interfere with the vehicle's overall control system and trigger malfunction conditions after installation or after a brief period of operation [17].

Another advantage is that unplanned repairs are reduced, and the severity of the fault can be determined. If the problem is limited to the information sensor, the vehicle can be put back into service and the problem can be fixed later within the scheduled downtime, which was impossible before $[18,19]$.

Unlike road cars, the DMU 812 series is not equipped with a blinking fault code performance; this means that without MAN-cats I, it is impossible to determine whether the rail vehicle is not running or whether the internal combustion engine has stopped.

The rail traffic schedule is disrupted, which has an indirect economic effect. When we replace diesel multiple units from the 812 series with locomotive 754 + heavy construction wagons or DMJ 861, it maximizes fuel consumption [20]. For comparison, the consumption of DMU 812 is 191 $\mathrm{g} \mathrm{kWh}^{-1}$, in the case of DMU 861, it is $199 \mathrm{~g} \mathrm{kWh}^{-1}, 224 \mathrm{~g} \mathrm{kWh}^{-1}$ for HKV 754. It reflects the following consumption values in terms of rated engine output and a diesel density of $0.84 \mathrm{~g} \mathrm{~cm}^{-3}$ :

- HDV 812 (MAN D 2866 LUH 21) ...................................... 58,4 $1 \mathrm{~h}^{-1}$

- DMJ 861 (MAN D 2842 LE 622) ..................................... 278,6 $1 \mathrm{~h}^{-1}$

- HKV 754 (ČKD K 12 V 230 DR)......................................389,3 $1 \mathrm{~h}^{-1}$

As a result of the above, replacing a DMU 812 series increases fuel consumption and hence running costs significantly $[11,21]$.

Currently, there are 186 MAN engines in operation in ZSSK a.s. Specific engine houses must be equipped with the most up-to-date edition of MAN-cats diagnosis for the purposes of repair.

Based on the previous short evaluation, it is obvious that purchasing MAN-cats III is financially beneficial (the price of the full MAN-cats III equipment was $€ 6,500$ in 2017, and the licensing fee was $€ 180$ a month). 


\section{Conclusion}

In practice, it was important to test whether the mentioned systems work in compliance with the enclosed instructions from individual component manufacturers. The technical requirements of the MAN D 2866 LUH 21 were discussed in the first chapter. In the second chapter, the time-based maintenance of MAN D 2866 LUH 21 was demonstrated, as well as the description of faults and fault signaling by MIREL 812. The third chapter describes how sensors in cable harnesses are connected and which of them provide information to MIREL 812. The following chapter focuses on fault detection and our tests on the MAN D 2866 LUH 21 engine using MAN-cats diagnosis. The paper provides a list of generated faults based on defining the sensors and their function in controlling the DMU 812 sequence. The paper also proposes a "fault decoder" based on collected data. The sixth chapter deals with the discussion on how MAN-cats I can significantly simplify and shorten repair procedures by detecting faults as well as the overall operation of the engine. Mechanics working at Fil'akovo engine house applied the above theoretical results in practice. Based on the results, diagnosis and troubleshooting on MAN popular rail engines can be managed using diagnosis MAN-cats III.

\section{Acknowledgments}

The research is supported by the Cultural and Educational Grant Agency of the Ministry of Education, Science, Research and Sport of the Slovak Republic under the project No. 045ŽU4/2021.

\section{References}

[1] Weis, P., Kucera, L., Pechac, P. \& Mocilan, M. (2017). Modal Analysis of Gearbox Housing with Applied Load. In: 12th International Scientific Conference of Young Scientists on Sustainable, Modern and Safe Transport, 31-Jun 02 May 2017, High Tatras, Slovakia. Book Series: Procedia Engineering, 192, 953-958. DOI: 10.1016/j.proeng.2017.06.164.

[2] Gramblicka, S., Kohar, R. \& Madaj, R. (2017). Construction Design Automatically Adjustable Mechanism for Crane Forks. In: 58th International Conference of Machine Design Departments (ICMD 2017), 06-08 September 2017, (pp. 100-103). Prague, Czech Republic: Czech University Life Sciences, Faculty of Engineering. ISBN 978-80-213-2769-6.

[3] Kohar, R., Stopka, M., Weis, P., Spisak, P. \& Steininger, J. (2020). Modular 3D Printer Concept. In: 59th International Conference of Machine Design Departments (ICMD 2018), 11-14 September 2018 (pp. 483-488). Demanovska Dolina, Slovakia. Book Series: Lecture Notes in Mechanical Engineering. ISBN 97830303314503. 
[4] Jankejech, P., Fabian, P., Broncek, J. \& Shalapko, Y. (2016). Influence of Tempering on Mechanical Properties of Induction Bents Below 540 Degrees C. Book Series: Acta Mechanica et Automatica 10(2), 81-86. DOI: 10.1515/ama-2016-0013.

[5] Jambor, M., Kajanek, D., Fintova, S., Broncek, J., Hadzima, B., Guagliano, M. \& Bagherifard, S. (2021). Directing Surface Functions by Inducing Ordered and Irregular Morphologies at Single and Two-Tiered Length Scales. Book Series: Advanced Engineering Materials, 23(2). Article Number: 2001057. DOI: 10.1002/adem.202001057.

[6] Orman, L.J., Radek, N. \& Broncek, J. (2018). Sintered Mesh Layers for the Production of Efficient Phase - Change Heat Exchangers. Book Series: Terotechnology, 5, 189-193. DOI: $10.21741 / 9781945291814-33$.

[7] Kucera, L., Gajdac, I. \& Kamas, P. (2014). Computing and Design of Electric Vehicles. In: 54th International Conference of Machine-Design-Departments, 10-12 September 2013 (pp. 105-111). Hejnice, Czech Republic. Book Series: Modern methods of construction design. DOI: 10.1007/978-3-319-05203-8_15.

[8] Kucera, L., Patin, B., Gajdosik, T., Palencar, R., Palencar, J. \& Ujlaky, M. (2020). Application of Metrological Approaches in the Design of Calibration Equipment for Verification of Float Level Gauges. Book Series: Measurement Science Review, 20(5), 230235. DOI: $10.2478 / \mathrm{msr}-2020-0028$.

[9] Galbavy, M., Pitonak, J. \& Kucera, L. (2014). Powershift Differential Transmission with Three Flows of Power for Hybrid Vehicles. In: 54th International Conference of MachineDesign-Departments, 10-12 September 2013 (pp. 27-33). Hejnice, Czech Republic. Book Series: Modern methods of construction design. DOI: 10.1007/978-3-319-05203-8_4.

[10] Broncek, J., Drbul, M., Stupavsky, M. \& Radek, N. (2017). The Application of New Rules of GPS in Structural Product Requirement. In: 58th International Conference of Machine Design Departments (ICMD), 06-08 September 2017 (pp. 50-55). Prague, Czech Republic: Czech University Life Sciences, Faculty of Engineering. ISBN 978-80-213-2769-6.

[11] Caco, M., Tribula, R., Scerba, P. \& Kohar, R. (2017). Application of Simulation Software to Optimize Construction Nodes of Ultrasonic Welding Machines. In 58th International Conference of Machine Design Departments (ICMD 2017), 06-08 September 2017 (pp. 5659). Prague, Czech Republic: Czech University Life Sciences, Faculty of Engineering. ISBN 978-80-213-2769-6.

[12] Sarkan, B., Kuranc, A. \& Kucera, L. (2019). Calculations of Exhaust Emissions Produced by Vehicle with Petrol Engine in Urban Area. In: 4th International Conference of Computational Methods in Engineering Science (CMES'19), 21-23 November 2019, Kazimierz Dolny, 
Poland. Book Series: IOP Conference Series-Materials Science and Engineering, 710. Article Number: 012023. ISBN 9781713810391.

[13] Liptakova, T., Broncek, J., Lovisek, M. \& Lago, J. (2017). Tribological and Corrosion Properties of Al-brass. In: 33rd Danubia Adria Symposium on Advances in Experimental Mechanics (DAS), 20-23 September 2016 (pp. 5867-5871). Portoroz, Slovenia. Book Series: Materials today-proceedings, 4(5). ISBN 9781713810391.

[14] Drbul, M., Martikan, P., Broncek, J., Litvaj, I. \& Svobodova, J. (2018). Analysis of Roughness Profile on Curved Surfaces. Book Series: Innovative Technologies in Engineering Production (ITEP'18), 244. Article Number: 01024. DOI: 10.1051/matecconf/201824401024.

[15] Majchrak, M., Kohar, R., Kajan, J. \& Skyba, R. (2019). 3D Meshing Methods of Ball-Rolling Bearings. In: 13th International Scientific Conference of Young Scientists on Sustainable, Modern and Safe Transport, 29-31 May 2019, Novy Smokovec, Slovakia. Book Series: Transportation Research Procedia, 40, 784-791. DOI: 10.1016/j.trpro.2019.07.111.

[16] Gramblicka, S., Kohar, R., Majchrak, M. \& Vrabec, M. (2020). Contact Analysis of Selected Toothed Contact of the Two-Stage Front Gearbox. In: 59th International Conference of Machine Design Departments (ICMD 2018), 11-14 September 2018 (pp. 263-269). Demanovska Dolina, Slovakia. Book Series: Lecture Notes in Mechanical Engineering. ISBN 9783030331450 .

[17] Rybicka, I., Droździel, P., Stopka, O. \& Lupták, V. 2018, "Methodology to propose a regional transport organization within specific integrated transport system: A case study", Transport Problems 13(4), 115-125. DOI: 10.20858/tp.2018.13.4.11.

[18] Chaba, R. (2021). Influence of telematics of ubi insurance on the management of the fleet of company vehicles. The Archives of Automotive Engineering - Archiwum Motoryzacji 92(2), 69-82. DOI: 10.14669/AM.VOL92.ART5.

[19] Iwnicki, S., Spiryagin, M., Cole, C. \& McSweeney, T (2020). Handbook of Railway Vehicle Dynamics. Second Edition. Boca Raton: CRC Press/Taylor \& Francis Group. ISBN 978-11386-0285-4.

[20] Lupták, V., Stopka, O. \& Jeřábek, K. (2017). "Draft deployment of traction units with active tilting system for regional and long-distance transport on non-modernized railway tracks", MATEC Web of Conferences. DOI:10.1051/matecconf/201713400034.

[21] Kucera, L. \& Gajdosik, T. (2014). The Vibrodiagnosis of Gears. In: 54th International Conference of Machine-Design-Departments, 10-12 September 2013 (pp. 113-118). Hejnice, Czech Republic. Book Series: Modern methods of construction design. DOI: 10.1007/978-3319-05203-8_16. 